\title{
Color screening in a constituent quark model of hadronic matter
}

\author{
G. Toledo Sáncheq* \\ Instituto de Física UNAM, A. P. 20-364 México 01000 D. F. México \\ J. Piekarewict 才 \\ Department of Physics, Florida State University, Tallahassee, FL 32306-4350
}

(Dated: September 5, 2018)

\begin{abstract}
The effect of color screening on the formation of a heavy quark-antiquark $(Q \bar{Q})$ bound statesuch as the $J / \psi$ meson - is studied using a constituent-quark model. The response of the nuclear medium to the addition of two color charges is simulated directly in terms of its quark constituents via a string-flip potential that allows for quark confinement within hadrons yet enables the hadrons to separate without generating unphysical long-range forces. Medium modifications to the properties of the heavy meson, such as its energy and its mean-square radius, are extracted by solving Schrödinger's equation for the $Q \bar{Q}$ pair in the presence of a (screened) density-dependent potential. The density dependence of the heavy-quark potential is in qualitative agreement with earlier studies of its temperature dependence extracted from lattice calculations at finite temperature. In the present model it is confirmed that abrupt changes in the properties of the $J / \psi$-meson in the hadronic medium (plasma), correlate strongly with the deconfining phase transition.
\end{abstract}

PACS numbers: 24.85.+p, 24.10.Lx, 25.75.Nq, 12.39.Jh

\section{INTRODUCTION}

The quest for novel states of matter remains a central theme in physics and one that spans all of its subfields. The discovery of high-temperature superconductors and the experimental realization of Bose-Einstein condensation are some recent examples. The widespread impact of these discoveries in the areas of condensed-matter physics and atomic physics is well documented in the literature. At the interface between nuclear and particle physicsbut with impact in cosmology and astrophysics - is the search for the quark-gluon plasma (QGP), a novel state of matter that may have existed at the dawn of the Universe and that may still exist today in the dense environments of exotic stars. The quark-gluon plasma, a deconfined state of quarks and gluons, is predicted from QCD to be attained at high temperatures and/or high baryon densities. A variety of experiments have been devoted to produce the QGP in the laboratory. These efforts started at the AGS in Brookhaven and at the SPS in CERN, and now continue at the relativistic-heavy-ion collider (RHIC), and should culminate with the construction of the large-hadron-collider (LHC) at CERN 1]. By colliding extremely energetic heavy ions, the aim of these experiments is to create a region of such high-energy density (or high temperature) that quarks and gluons will become deconfined. While current experimental facilities (primarily RHIC) may have already created the QGP [2], a great challenge remains: how to identify clearly and unambiguously the production of such coveted state?

Spatial observations complement terrestrial searches

\footnotetext{
*Electronic address: toledo@fisica.unam.mx
}

${ }^{\dagger}$ Electronic address: jorgep@csit.fsu.edu for the QGP. The advent of sophisticated telescopes operating at a variety of wavelengths have turned neutron stars from theoretical curiosities into powerful diagnostic tools. The observation of an anomalous mass-radius relation and/or an enhanced cooling in neutron stars, may provide strong evidence in support of strange stars [3], quark stars 4], or neutron stars with exotic cores. These cores may contain novel states of matter, such as meson condensates, strange-quark matter, and/or color superconductors [5, 6, 7].

In the case of terrestrial searches for the QGP, there is a set of complementary observables which provide information at different stages of the experiment. For example, leptons and photons produced at the center of the collision are expected to carry valuable information concerning the earliest stages of the collisions, while the relative abundance of light-flavor hadrons should reflect the freeze-out stage. Evidence for the QGP is also imprinted in the response of the medium to the formation and propagation of a heavy quark-antiquark pair, where Debye screening of the bound state $(e . g ., J / \psi)$ is expected [8, 9]. In this case it is important to study the possible dissolution of the bound state and the evolution of the confining potential with baryon and/or energy density. While the quest for these experimental signatures will undoubtedly continue, convincing arguments in favor of the discovery of a strongly-coupled QGP at RHIC have recently been made [10]. This persuasive study, containing a host of valuable references, uses existing observables - such as bulk collective flow and jet quenching - to justify the claim.

In the present work we study the efficiency of the nuclear medium in screening the color charges of a heavy quark-antiquark pair. That is, we study color screening as a function of the baryon density rather than as a function of temperature. This distinction is important 
as our $T=0$ formalism is unable to shed light on finitetemperature calculations, which themselves appear to be in a state of flux. Calculations based on potential models predict the $J / \psi$-meson to dissolve at a temperature of $T \simeq 1.1 T_{\mathrm{c}}[11]$ (with $T_{\mathrm{c}}$ being the critical temperature for the deconfining phase transition). However, recent lattice results seem to suggest that the $J / \psi$-meson survives up to a temperature of $T \simeq 1.5 T_{\mathrm{c}}[12,13]$. Moreover, the originally strong suppression of the $J / \psi$-meson predicted by Matsui and Satz has been recently put into question. Indeed, it has been argued that this elusive suppression may be offset (at least in part) by statistical recombination of $J / \psi$-mesons from the initially produced $c \bar{c}$ pairs 14, 15, 16, 17]. We offer no new insights into these important problems. Rather, we consider a simple model that dynamically interpolates between a hadronbased description at low-density and a quark-based description at high-density to study the dynamics of the $J / \psi$-meson. In essence, we aim at computing a heavyquark potential as a function of baryon density to complement (and compare to) lattice studies 18, 19, 20 that, while successful at finite-temperature, are unable to simulate hadronic systems at finite baryon density.

To correlate the nuclear-to-quark-matter transition to the in-medium properties of the $J / \psi$ meson, a string-flip model is used 21]. Although we will refer explicitly to the $\mathrm{J} / \psi$ meson henceforth, our approach is applicable to any heavy meson (or baryon) added to the hadronic medium. The string-flip model belongs to a class of constituentquark models that incorporates many of the phenomenological features of quantum chromodynamics (QCD). The underlying degrees of freedom are constituent quarks that interact via confining strings (or flux tubes) that arrange themselves instantaneously to the optimal configuration of quarks 22, 23, 24, 25]. The string-flip model is thus reminiscent of the Born-Oppenheimer approximation of wide use in atomic physics where the fast degrees of freedom (electrons or gluons) adjust instantaneously to changes in the slow degrees of freedom (ions and constituent quarks). The cornerstone of the string-flip model is a many-body potential that: a) is symmetric under the exchange of identical quarks, b) confines quarks within color-singlet clusters, and c) enables these clusters to separate without generating unphysical (long-range) van der Waals forces [26]. We regard these as a minimal characterization of any realistic quark-based model of hadronic matter. To our knowledge, there is no "conventional" potential, namely, one that may be written as a sum of two-body (or even three-body) forces, that satisfies the above requirements. Thus, the string-flip potential is truly many body; moving a single quark may affect the interaction among all the quarks in the system. The many-body potential is obtained by solving a difficult optimization problem [27], as one must decide how to assign colored quarks into color-singlet clusters. This "quarkassignment" problem is meant to represent the optimal configuration of gluonic strings.

The manuscript has been organized as follows. In
Sec. IIwe define the string-flip potential and discuss some of its most important characteristics. Further, we introduce a one-parameter variational wave function that, in spite of its simplicity, is exact in the low- and high-density limits. Indeed, at low density the variational wave function favors a strong clustering of quarks into color-singlet objects ("baryons"), while in the high-density limit only Pauli correlations remain. Finally, medium modifications to the properties of a heavy meson are addressed via a density-dependent heavy-quark potential. In Sec. III we present our results paying special emphasis to the correlations between the medium modifications to the properties of the $J / \psi$-meson and the deconfining phase transition. A summary and conclusions are presented in Sec. IV

\section{FORMALISM}

An exact many-body treatment of QCD at finite baryon density remains an unsolved problem. Although fresh new ideas continue to emerge, we use a $Q C D$ inspired model to study the screening of color charge by the nuclear medium. The main feature of the stringflip model employed here is an adiabatic many-body potential characterized by the arrangement of the gluonic strings (or flux-tubes) into a minimum-energy configuration [21, 22]. Such a many-body potential is symmetric under the exchange of identical quarks and is able to confine quarks within color-singlet clusters ("baryons"). Yet the confining force saturates within a baryon, thereby allowing the clusters to separate without generating unobserved long-range van der Waals forces [26]. While the exact functional form of the potential remains uncertain, the simultaneous requirements of quark confinement and cluster separability are likely to require solving some type of quark-assignment problem: which quarks belong to which color-singlet cluster? In what follows, the most important features of the string-flip model will be addressed. A more thorough discussion may be found in Ref. [28].

\section{A. The string-flip potential}

Following a choice introduced in earlier references 25, 28], one starts by defining the optimal pairing between red and blue quarks as follows:

$$
V_{R B}=\min _{P} \sum_{i=1}^{A} v\left(\mathbf{r}_{i R}, \mathbf{r}_{j B}\right),
$$

where $\mathbf{r}_{i R}$ denotes the spatial coordinate of the $i t h$ red quark and $\mathbf{r}_{j B}$ is the coordinate of its blue-quark "partner" $(i B \mapsto P(i B) \equiv j B)$. Note that the minimization procedure is over all possible $A$ ! permutations of the $A$ blue quarks and that the confining potential $v$ is assumed 
harmonic with a spring constant denoted by $k$. That is,

$$
v\left(\mathbf{r}_{i R}, \mathbf{r}_{j B}\right)=\frac{1}{2} k\left(\mathbf{r}_{i R}-\mathbf{r}_{j B}\right)^{2} .
$$

The "blue-green" and "green-red" components of the many-quark potential are defined in direct analogy to Eq. (11)

$$
\begin{aligned}
& V_{B G}=\min _{P} \sum_{i=1}^{A} v\left(\mathbf{r}_{i B}, \mathbf{r}_{j G}\right) \\
& V_{G R}=\min _{P} \sum_{i=1}^{A} v\left(\mathbf{r}_{i G}, \mathbf{r}_{j R}\right) .
\end{aligned}
$$

In this way, the many-body potential of the system is obtained by simply adding the three different pairwise contributions

$$
V\left(\mathbf{r}_{1}, \ldots, \mathbf{r}_{N}\right)=V_{R B}+V_{B G}+V_{G R} .
$$

The many-body Hamiltonian describing a system of $N$ quarks each with mass $m$ and momentum $\mathbf{p}_{i}$ is now given by

$$
H=\sum_{i=1}^{N} \frac{\mathbf{p}_{i}^{2}}{2 m}+V\left(\mathbf{r}_{1}, \ldots, \mathbf{r}_{N}\right),
$$

Note that the many-body potential defined in Eq. (4) is flavor blind. That is, the optimal pairing among the quarks is done according to their color but not their flavor. In practice, one may imagine collecting all quarks into red and blue "buckets" according to their color - but irrespective of their flavor - and then searching for their optimal pairing. This is then repeated for blue/green and green/red quarks. In the course of this work we adopt units in which $k=m=1$.

\section{B. The variational wave function}

The complicated many-body dynamics may be captured with a simple one-parameter variational wave function of the following form:

$$
\Psi_{\lambda}\left(\mathbf{r}_{1}, \ldots, \mathbf{r}_{N}\right)=e^{-\lambda V\left(\mathbf{r}_{1}, \ldots, \mathbf{r}_{N}\right)} \Phi_{F G}\left(\mathbf{r}_{1}, \ldots, \mathbf{r}_{N}\right) .
$$

Here $\lambda$ is the sole variational parameter, $V$ is the manybody potential defined in Eq. (4), and $\Phi_{F G}$ is a Fermigas wave function consisting of a product of Slater determinants. The variational parameter may be regarded as the order parameter for the nuclear-to-quark-matter transition, as $\lambda^{-1 / 2}$ represents the length-scale for quark confinement. Indeed, at low density the average interquark separation is much larger than the confining scale $\left(\lambda^{-1 / 2} \simeq 1\right)$ and the clustering of three quarks into colorsinglet clusters (nucleons) ensues. Note that while the interactions between quarks within a single nucleon are strong, the residual nucleon-nucleon interaction is weak, as the color force saturates within each individual nucleon. This many-body feature of the model precludes the development of long-range van der Waals forces. As the density increases, the average inter-quark separation will become comparable to the confining scale. This will signal the transition to the quark Fermi-gas phase. In the high-density regime the interactions between quarks are weak (asymptotic freedom) and the system "dissolves" into a free Fermi gas of quarks. No correlations between quarks remain, except those induced by the Pauli exclusion principle. Further details on the variational wave function may be found in Ref. 28]. For reference, the value of the variational parameter for an isolated nucleon is given by $\lambda_{0}=1 / \sqrt{3}$

\section{Variational Monte Carlo}

The structure of the variational wave function entails an important simplification, as the expectation value of the kinetic energy, which involves derivatives, may be simplified through an integration by parts. This yields [28],

$$
\left\langle\Psi_{\lambda}|T| \Psi_{\lambda}\right\rangle \equiv\langle T\rangle_{\lambda}=T_{F G}+2 \lambda^{2}\langle W\rangle_{\lambda} .
$$

Here $T_{F G}$ is the kinetic energy of a $(\lambda=0)$ free Fermi gas and $\langle W\rangle_{\lambda}$ is given by

$$
W=\sum_{n=1}^{N} \frac{1}{m}\left(\mathbf{x}_{n}-\mathbf{y}_{n}\right)^{2},
$$

where the sum is over all quarks in the system and $\mathbf{y}_{n}$ represents the average position of the two quarks connected to the $n_{\text {th }}$ quark located at $\mathbf{x}_{n}$. The increase in the kinetic energy of the system relative to that of a free Fermi gas is the result of clustering correlations. Of course, this "penalty" is more than compensated by the potential energy which favors the clustering of quarks into nucleons at low densities. The expectation value of the total energy of the system now reduces to the following expression:

$$
E(\lambda)=T_{F G}+2 \lambda^{2}\langle W\rangle_{\lambda}+\langle V\rangle_{\lambda} .
$$

This form is particularly convenient because the two functions that remain to be evaluated $(V$ and $W$ ) are local and may therefore be computed using standard Monte-Carlo techniques [29].

\section{The heavy-quark potential}

In this section we introduce the heavy-quark potential to correlate the modifications of the in-medium properties of the $J / \psi$-meson to the deconfinement phase transition. The heavy-quark potential is constructed as follows. Consider the original system of $N$ quarks described by a variational wave function as in Eq. (6). We assume that the variational parameter $\lambda$ has been fixed at its optimal 
value. After a large number of thermalization sweeps, one measures the expectation value of the potential energy [Eq. (4)]

$$
\langle V\rangle_{A}=\lim _{M \rightarrow \infty} \frac{1}{M} \sum_{m=1}^{M} V\left(\mathbf{r}_{1}^{(m)}, \ldots, \mathbf{r}_{N}^{(m)}\right),
$$

where the $M$ configurations of quarks are distributed according to the square of the variational wave function. At the same time that one pauses the Monte Carlo procedure to measure the potential energy in the $m_{\text {th }}$ configuration, one introduces a heavy quark-antiquark (e.g., $c \bar{c})$ pair separated by a fixed distance $r$. This procedure is implemented in our simulations by adding a triplet of static quarks to the system; red and blue quarks fixed at the origin and a green quark fixed a distance $r$ away. The red and blue quarks are fixed at the same location to simulate a source of anti-green color. As the heavy quarks are assumed static, their masses play no role in the simulations. Having added the three heavy quarks into the system, one now evaluates the potential energy of the $(A+1)$-body system by re-computing the optimal pairing of quarks into hadrons. (Note that the added red and blue heavy quarks are constrained to pair with each other.) That is,

$$
\langle V\rangle_{A+1}(r)=\lim _{M \rightarrow \infty} \frac{1}{M} \sum_{m=1}^{M} V\left(\mathbf{r}_{1}^{(m)}, \ldots, \mathbf{r}_{N}^{(m)} ; r\right)
$$

The heavy-quark potential is defined as the difference between the potential energy of the $(A+1)$-body system relative to that of the $A$-body system:

$$
V_{Q \bar{Q}}(r)=\langle V\rangle_{A+1}(r)-\langle V\rangle_{A} .
$$

Note that the heavy-quark potential is computed in the sudden approximation. That is, the many-quark wave function [Eq. [6)] (and thus the location of all the light quarks in the system) is assumed to remain unchanged as the heavy quarks are introduced into the system. Not so, however, the pairing. The presence of the medium screens the interaction between the heavy quarks by finding the optimal pairing of the $N=3(A+1)$ quarks into hadrons. In this way, the potential energy between the $Q \bar{Q}$ pair gets modified relative to its free-space form because of the screening. In general, $V_{Q \bar{Q}}(r) \neq k r^{2}$.

\section{E. The $J / \psi$ meson in free space}

To quantify how a $J / \psi$-meson (or a comparable heavy meson) is modified by color screening, we review its freespace properties in a model with harmonic confinement. Consider a $J / \psi$-meson as a nonrelativistic system of a quark-antiquark $(c \bar{c})$ pair of mass $m_{c}$ interacting via a harmonic confining potential of spring constant $2 k$. That is,

$$
H=\frac{\mathbf{p}_{1}^{2}}{2 m_{c}}+\frac{\mathbf{p}_{2}^{2}}{2 m_{c}}+\frac{1}{2}(2 k)\left(\mathbf{r}_{1}-\mathbf{r}_{2}\right)^{2} .
$$

Introducing center-of-mass and relative coordinates

$$
\mathbf{R}=\frac{1}{2}\left(\mathbf{r}_{1}+\mathbf{r}_{2}\right), \quad \mathbf{r}=\left(\mathbf{r}_{1}-\mathbf{r}_{2}\right)
$$

enables one to reduce the above Hamiltonian to the following simple form:

$$
H=\frac{P^{2}}{4 m_{c}}+\left(\frac{p^{2}}{m_{c}}+k \mathbf{r}^{2}\right) .
$$

Describing the motion of the system relative to its center of mass, the ground-state wave function of the $J / \psi$ meson in the present harmonic approximation becomes

$$
\Phi_{J / \psi}(\mathbf{r})=\frac{e^{-r^{2} / 2 b^{2}}}{\left(\pi b^{2}\right)^{3 / 4}}\left|\chi_{c} \chi_{\bar{c}}\right\rangle_{1 M}, \quad b \equiv\left(k m_{c}\right)^{-1 / 4} .
$$

Here $b$ is the harmonic-oscillator length and $\chi$ represents a spin- $1 / 2$ spinor. Using the above wave function, the ground-state energy and mean-square radius of the $J / \psi$ meson in free space are given by

$$
\begin{aligned}
& E_{J / \psi}=2 m_{c}+\frac{3}{2} \sqrt{\frac{4 k}{m_{c}}} \longrightarrow 11.342, \\
& r_{J / \psi}^{2}=\frac{3}{8} \frac{1}{\sqrt{k m_{c}}} \longrightarrow 0.168 .
\end{aligned}
$$

The mean-square radius has been obtained by evaluating the ground-state expectation value of the operator $r^{2} / 4$. Moreover, the arrows in Eq. (17) indicate the appropriate numerical values for the energy and the mean-square radius in units in which $k=1$ and $m_{c} / m=5$. Note that the mass of the charm quark $(\simeq 1.5 \mathrm{GeV})$ has been fixed at five times the value of the mass of the light constituent quarks $(\simeq 0.3 \mathrm{GeV})$. Our goal now is to quantify how these baseline values change as the density of the medium increases and, further, to correlate these changes to the deconfining phase transition.

\section{RESULTS}

Variational Monte-Carlo calculations of a large number of ground-state observables, such as the energy, the two-body correlation function, and the strangeness-perbaryon ratio have been reported in an earlier publication [28]. Here we borrow values for the optimized variational parameter to compute the heavy-quark potential as indicated in Eq. (12). Results obtained from these simulations are displayed in Fig. 11 for a variety of densities around the deconfining transition density of $\rho_{\mathrm{c}} \simeq 0.09$. Recall that the density is given in dimensionless units (for a conversion to physical units see Ref. [28]). The symbols in Fig. 1] denote the outcome from the MonteCarlo simulations while the lines represent analytic fits to the simulation data. While it is evident that quantitative changes develop below the critical density as a result 
of screening, no major qualitative changes emerge. For example, the $Q \bar{Q}$ potential remains confining for all densities below the critical density $\rho_{\mathrm{c}}$. However, at densities above $\rho_{\mathrm{c}}$, a dramatic change in the shape of the potential emerges. Indeed, the heavy-quark potential ceases to be confining and a $Q \bar{Q}$ spectrum supporting both bound and continuum states develops. This behavior is reminiscent of the temperature dependence of the heavy-quark potential displayed in Fig. 6 of Ref. [18].

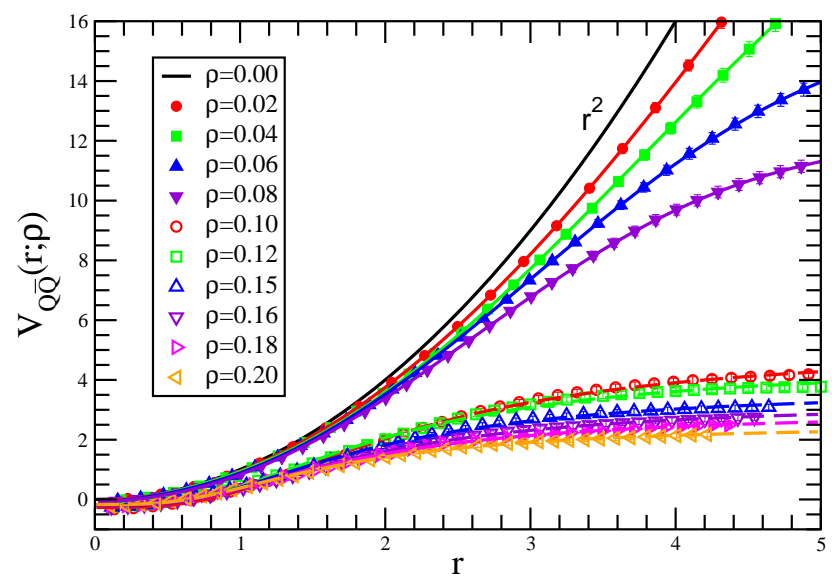

FIG. 1: Density dependence of the heavy-quark potential as a function of the separation $r$ between the heavy-quark pair. The $\rho=0$ potential, $V_{Q \bar{Q}}(r)=r^{2}$, represents the baseline (freespace) potential.

Medium modifications to the properties of the $J / \psi$ meson are studied by solving Schrödinger's equation in the presence of the heavy-quark potential. Recall that we have fixed the mass of the charm quark (in units of the light-quark constituent mass) to $m_{c} / m=5$. Expressing the "reduced" $J / \psi$ wave function as

$$
\Phi_{J / \psi}(\mathbf{r})=\frac{\varphi(r)}{r}\left|\chi_{c} \chi_{\bar{c}}\right\rangle_{1 M}
$$

the Schrödinger equation for the ground-state $(l=0)$ wave function takes the following simple form:

$$
\left(-\frac{\hbar^{2}}{2 \mu_{c}} \frac{d^{2}}{d r^{2}}+V_{Q \bar{Q}}(r ; \rho)\right) \varphi(r)=E \varphi(r) .
$$

Here $\mu_{c} \equiv m_{c} / 2$ is the reduced mass of the $c \bar{c}$ pair. In Fig. 2 we display the ground-state density $\varphi^{2}(r)$ weighted by $r^{2} / 4$, so that the area under the curve yields directly the mean-square radius of the $J / \psi$-meson, which we have enclosed in brackets. For densities below the transition, the wave functions cluster around the free-space value. Indeed, the change in the mean-square radius of the $J / \psi$ meson between $\rho=0$ and $\rho=0.08$ amounts to less than $6 \%$. However, as soon as the transition density is crossed, a significant spread in the wave function develops, resulting in both a reduced binding energy and an increased mean-square radius (see Fig. 3). For comparison, at a density just above the transition $(\rho=0.10)$, the energy decreases by almost $50 \%$ and the mean-square radius increases by close to $25 \%$, relative to their free-space values.

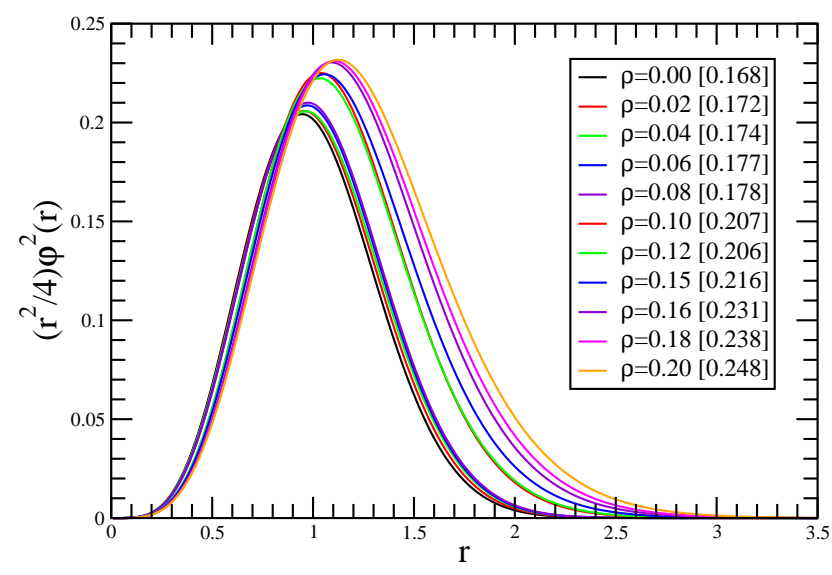

FIG. 2: Ground-state densities for the $J / \psi$ meson weighted by $r^{2} / 4$ for a variety of densities. The quantities in brackets are the corresponding mean-square radii, which are obtained by integrating the area under the curves.

Finally, we use Fig. 3] to correlate modifications to the properties of the $J / \psi$-meson in the medium to the deconfining phase transition. Note that all shown quantities are given in units of their free-space values. The density dependence of the variational parameter $\lambda$ exhibits an abrupt drop at a quark density of about $\rho=0.09$. As $\lambda^{-1 / 2}$ represents the length-scale for quark confinement, this drop signals the onset of the transition to quark matter. Recall that for $\lambda \equiv 0$ the only remaining correlations in the many-quark wave function are due to the Pauliexclusion principle [see Eq. [6] ]. While smaller in magnitude, the corresponding abrupt changes in the energy and mean-square radius of the $J / \psi$ are unmistakable. This provides confirmation of the long-time paradigm that modifications to the properties of the $J / \psi$-meson in the medium represents a robust indicator of the deconfining phase transition. This in a model that, while simple, uses exclusively quark degrees of freedom to describe dynamically the deconfining phase transition.

\section{CONCLUSIONS}

Modification to the properties of the $J / \psi$ meson in the nuclear medium were computed in a constituent quark model of hadronic matter, that confines quarks within individual color-neutral clusters, yet allows the clusters to separate without generating unphysical longrange forces. One of the greatest virtues of this string-flip 


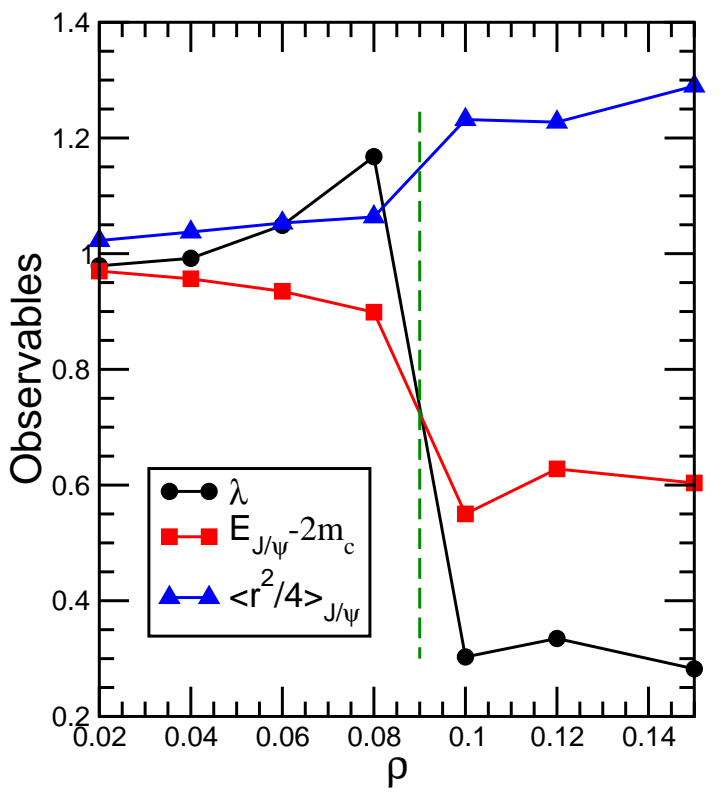

FIG. 3: Density dependence of the energy and mean-square radius of the $J / \psi$ meson. The "jump" in these quantities at $\rho \simeq 0.09$ is correlated to the corresponding jump in the variational parameter $\lambda$ at the onset of the nuclear-to-quark matter transition. All quantities are measured in units of their free-space values.

model is the description of the deconfining phase transition. Indeed, the evolution of the system from a nuclear phase, in which quarks clusters into color-singlet hadrons, to a quark Fermi-gas phase is dynamical in the model; there is no need for additional ad-hoc parameters to characterize the transition.

To quantify how the properties of the $J / \psi$ meson get modified by color screening we computed the heavy- quark potential. Operationally, this was effected by adding two heavy sources of color charge into the system and monitoring how the interaction between the charges gets modified by the presence of the medium. Screening of the heavy-quark charges by the medium yields an effective potential that is drastically different from its free-space value above the transition density. Although the potential below the transition is also modified by the medium, it retains its basic form. In contrast, above the transition density the potential ceases to be confining and a "heavy quarkonium" spectrum that supports both bound and continuum states develops.

In our model one of the main indicators ("the order parameter") of the phase transition is the variational parameter of the many-quark wave function. This parameter, which represents the length-scale for quark confinement, drops abruptly to (almost) zero at the transition density indicating how clustering correlations cease to be important as the system evolves into the quarkmatter phase. This drop correlates strongly to similar abrupt changes in the in-medium properties of the $J / \psi$ meson. This correlation confirms - in a model that uses exclusively quarks and flux tubes (or strings) as the fundamental degree of freedom - the long-standing tenet of Matsui and Satz [8] of using medium modifications to the $J / \psi$-meson as a robust signature for the onset of the deconfining phase transition.

\section{Acknowledgments}

We thank Prof. Anthony Frawley for many useful conversations. This work was supported in part by the United State Department of Energy under Contract No.DE-FG05-92ER40750 and by Conacyt México under grants 41600-A1, 41048-A1 and 42026-F.
[1] G. Baym, Nucl. Phys. A698, 23 (2002), hep-ph/0104138.

[2] S. S. Adler and et al., Phys. Rev. C 69, 014901 (2004), nucl-ex/0305030.

[3] R. X. Xu, ApJ 570, L65 (2002).

[4] J. J. Drake and et al., ApJ 572, 996 (2002).

[5] G. Baym and C. Pethick, Ann. Rev. Astron. Astrophys. 17, 415 (1979).

[6] E. Witten, Phys. Rev. D 30, 272 (1984).

[7] M. G. Alford, K. Rajagopal, and F. Wilczek, Phys. Lett. B422, 247 (1998), hep-ph/9711395.

[8] T. Matsui and H. Satz, Phys. Lett. B178, 416 (1986).

[9] T. Matsui, Prog. Theor. Phys. Suppl. 151, 11 (2003), nucl-th/0305096.

[10] M. Gyulassy (2004), nucl-th/0403032.

[11] S. Digal and et al., Phys. Rev. D 64, 094015 (2001).

[12] S. Datta, F. Karsch, P. Petreczky, and I. Wetzorke, Nucl. Phys. B 119, 487 (2003).

[13] S. Datta, F. Karsch, P. Petreczky, and I. Wetzorke (2003), hep-lat/0312037.
[14] P. Braun-Munzinger and J. Stachel, Phys. Lett. B 490, 196 (2000), nucl-th/0007059.

[15] R. L. Thews, M. Schroedter, and J. Rafelski, Phys. Rev. C 63, 054905 (2001), hep-ph/0007323.

[16] A. Andronic, P. Braun-Munzinger, K. Redlich, and J. Stachel, Phys. Lett. B 571, 36 (2003), nuclth/0303036.

[17] R. L. Thews, Jour. Phys. G 30, S369 (2004), hepph/0305316.

[18] F. Karsch, E. Laermann, and A. Peikert, Nucl. Phys. B 605, 579 (2001), hep-lat/0012023.

[19] T. Umeda, H. Matsufuru, O. Miyamura, and K. Nomura, Nucl. Phys. A 721, 922 (2003), hep-lat/0212007.

[20] R. Rapp and L. Grandchamp, Jour. Phys. G 30, S305 (2004), hep-ph/0305143.

[21] F. Lenz, J. T. Londergan, E. Moniz, R. Rosenfelder, M. Stingl, and K. Yazaki, Ann. Phys. 170, 65 (1986).

[22] C. J. Horowitz, E. J. Moniz, and J. W. Negele, Phys. Rev. D 31, 1689 (1985). 
[23] P. J. S. Watson, Nucl. Phys. A494, 543 (1989).

[24] C. J. Horowitz and J. Piekarewicz, Phys. Rev. C 44, 2753 (1991).

[25] C. J. Horowitz and J. Piekarewicz, Nucl. Phys. A536, 669 (1992).

[26] O. W. Greenberg and L. H. J., Nucl. Phys. A370, 349 (1981).

[27] R. E. Murkard and U. Derigs, Lecture Notes in Eco- nomics and Mathematical Systems, vol. 184 (Springer, Berlin, 1990).

[28] G. Toledo Sanchez and J. Piekarewicz, Phys. Rev. C 65, 045208 (2002).

[29] N. Metropolis, A. Rosenbluth, M. Rosenbluth, A. Teller, and E. Teller, Jour. Chem Phys. 21, 1087 (1953). 